\title{
SNP rs2470152 in CYP19 is correlated to aromatase activity in Chinese polycystic ovary syndrome patients
}

\author{
XIN-LIN ZHANG ${ }^{1 *}$, CHENG-WEI ZHANG $^{1 *}$, PEI XU $^{1}$, FENG-JING LIANG $^{1}$, YE-NA CHE $^{1}$, YAN-JIE XIA $^{1}$, \\ YUN-XIA CAO ${ }^{2}$, XIAO-KE WU ${ }^{3}$, WEN-JUN WANG ${ }^{4}$, LONG YI $^{1}$, QIAN GAO $^{1}$ and YONG WANG ${ }^{1}$ \\ ${ }^{1}$ Center for Translational Medicine and Jiangsu Key Laboratory of Molecular Medicine, Medical School of Nanjing University, \\ Nanjing 210093; ${ }^{2}$ Department of Obstetrics and Gynecology, Anhui Medical University, Hefei 230022; \\ ${ }^{3}$ Department of Obstetrics and Gynecology, The First Affiliated Hospital, Heilongjiang University of Chinese Medicine, \\ Harbin 150040; ${ }^{4}$ Centre of Reproduction, Department of Obstetric and Gynaecology, \\ Memorial Hospital of Sun Yat-Sen University, Guangzhou 510120, P.R. China
}

Received July 24, 2011; Accepted September 28, 2011

DOI: $10.3892 / \mathrm{mmr} .2011 .616$

\begin{abstract}
CYP19 encodes aromatase, a key enzyme essential for estrogen biosynthesis. Single nucleotide polymorphism (SNP) rs2470152 in CYP19 is associated with serum estradiol (E2) level and the E2/T (estradiol/testosterone) ratio. A case-control study including 661 individuals [364 polycystic ovary syndrome (PCOS) patients and 297 controls] was conducted to assess the association of SNP rs2470152 with PCOS. The subjects were genotyped using the polymerase chain reaction-restriction fragment length polymorphism method. Hormone levels were analyzed among various genotypes. The genotypic distributions of rs 2470152 did not differ in PCOS patients when compared to the controls. However, differences in the $\mathrm{E} 2 / \mathrm{T}$ ratio were detected, exhibiting a lower ratio in the heterozygous $T C$ genotype in PCOS patients $(\mathrm{p}=0.01036)$ and controls $(p=0.000)$. Testosterone levels also differed between the three genotypes of PCOS patients $(p=0.00625)$, with a higher level in the TC genotype. Therefore, rs2470152 in CYP19 was not a major etiological factor for PCOS; however,
\end{abstract}

Correspondence to: Dr Yong Wang, Center for Translational Medicine and Jiangsu Key Laboratory of Molecular Medicine, Medical School of Nanjing University, Nanjing 210093, P.R. China E-mail: yongwang@nju.edu.cn

${ }^{*}$ Contributed equally

Abbreviations: PCOS, polycystic ovary syndrome; LH, luteinizing hormone; FSH, follicle-stimulating hormone; E2, estradiol; BMI, body mass index; AAM, age at menarche; T, testosterone; $\mathrm{P}$, progesterone; RIA, radioimmunity assay; $\mathrm{PCR}$, polymerase chain reaction; PCO, polycystic ovarian; GOOD, Gothenburg Osteoporosis and Obesity Determinants; MrOS, Osteoporotic Fractures in Men

Key words: polycystic ovary syndrome, CYP19, single nucleotide polymorphism, rs2470152, aromatase activity the heterozygous $T C$ genotype may inhibit aromatase activity, resulting in hyperandrogenism, particularly in PCOS patients.

\section{Introduction}

Polycystic ovary syndrome (PCOS) is one of the most controversial entities in gynecological endocrinology, with a prevalence of $7.4 \%$ in women of reproductive age in China (1). PCOS is characterized by the presence of hyperandrogenism, menstrual irregularities and, in a significant portion of patients, insulin resistance (2-4). The familial aggregation of PCOS, hyperandrogenism and associated metabolic abnormalities indicates a genetic origin for PCOS (5). Heterogeneous clinical manifestations influenced by ethnic factors indicate the involvement of environmental factors (6). To date, dozens of genes have been studied for association with PCOS, mostly using 'case-control study' and 'linkage analysis' methods. Genes investigated are mainly related to the regulation of androgen biosynthesis and function, insulin resistance and chronic inflammation, including CYP19 (7,8), CYP11A1 (9), CYP17 (10), HSD17B6 (11), the insulin gene (12) and TCF7L2 (13); however, currently no single gene has been universally accepted as the fundamental cause of PCOS, partially due to the fact that a globally accepted diagnostic scheme for PCOS is not available.

The enzyme complex aromatase is responsible for the conversion of C19 steroids (androgens) into C18 steroids (estrogens). This enzyme complex is composed of cytochrome $\mathrm{P} 450$ aromatase and NADPH cytochrome P450 reductase30. Aromtase is encoded by CYP19 located at $15 \mathrm{q} 21.1$ (14). As a key enzyme in the conversion of androgens to estrogens, aromatase may play a potential role in the development of hyperandrogenism. Aromatase deficiency has been reported in a number of hyperandrogenic patients (15). Recently, single nucleotide polymorphism (SNP) rs2414096 in an intron of CYP19 has been reported to be associated with susceptibility to PCOS in Chinese women, and the rs2414096 $A$ allele may be associated with the activity of aromatase (8). These observations indicate that altered regulation of aromatase may be involved in PCOS. 
SNP rs2470152 has been reported to be associated with serum estrogen levels and the E2/T (estradiol/testosterone) ratio $(16,17)$. In this study, we investigated whether SNP rs2470152 is associated with susceptibility to PCOS by designing a case-control experiment, including 364 PCOS patients and 297 non-PCOS individuals as controls.

\section{Materials and methods}

Subjects. A total of 661 Han Chinese women were included in our study. Among them, 364 were patients with PCOS and 297 were non-PCOS controls. The PCOS patients were diagnosed based on the 2003 Rotterdam Criteria. Controls had normal ovulatory menstrual cycles without hirsutism and other manifestations of hyperandrogenism. None of the controls exhibited obesity or insulin resistance. Peripheral blood samples were collected in Nanjing Drum Tower Hospital, the Affiliated Hospital of Medical School of Nanjing University or at the Department of Obstetrics and Gynecology, Anhui Medical University, between 2004 and 2010. Serum steroid levels of 275 PCOS patients and 235 control women were available for analysis. The study was approved by the Medical School of Nanjing University, and informed consent was obtained from the women prior to inclusion.

PCOS diagnostic criteria and hormone measurements. Patients with PCOS were diagnosed according to the 2003 Rotterdam Criteria (18) (The Rotterdam ESHRE/ASRM-Sponsored PCOS Consensus Workshop Group, 2004), as previously described (19).

Clinical variables, such as body weight and height, were assessed and body mass index (BMI) was calculated as body weight in kilograms divided by the square of the height in meters. Age at menarche (AAM) was obtained through inquiry. Venous blood samples were collected during the 3 rd to the 5 th day of the menstrual cycle for those who had menstruation and at any time for those who had amenorrhea. Samples were collected between 08:00 and 09:00 after a 12-h overnight fast. Blood samples were immediately centrifuged and serum was separated and frozen at $-80^{\circ} \mathrm{C}$ until assayed. None of the study participants had been receiving oral contraceptives or drug therapy for the 3 months prior to hormone measurement. Levels of total testosterone (T), estradiol (E2), follicle-stimulating hormone (FSH), luteinizing hormone ( $\mathrm{LH}$ ) and prolactin (PRL) in the sera were detected by RIA (Beijing North Institute of Biological Technology of China and the CIS Company of France). Intra- and inter-assay coefficients of variation were $<10 \%$ for all the assays.

Genotype analysis of polymorphisms. Genomic DNA was extracted from human peripheral blood samples using an SBS UltraPure $^{\mathrm{TM}}$ Genome DNA kit (SBS Genetech, Shanghai, China) and stored at $-20^{\circ} \mathrm{C}$. All subjects were genotyped using the polymerase chain reaction-restriction fragment length polymorphism method. PCR primers for the fragment containing the SNP were forward 5'-CTG CCT TTG AGG AGC TTA CTG T-3' and reverse 5'-CTT CTC TGG CTT TCC CCT CT-3'. PCR amplification was conducted in a total volume of $25 \mu \mathrm{l}$ containing $50 \mathrm{ng}$ of genomic DNA, $6.25 \mathrm{pmol}$ of each primer, $2.5 \mu \mathrm{l}$ short tandem repeat (STR) 10X buffer (STR
10X buffer; Promega, Madison, WI, USA) and 0.75 units of GoTaq DNA polymerase (Promega). The PCR was performed with a TaKaRa PCR Thermal Cycler Dice (TP600; Takara Bio Inc., Japan). The PCR conditions were as follows: $96^{\circ} \mathrm{C}$ denaturing for $2 \mathrm{~min}$, followed by 30 cycles consisting of $1 \mathrm{~min}$ of denaturation at $94^{\circ} \mathrm{C}, 1 \mathrm{~min}$ of annealing at $60^{\circ} \mathrm{C}$ and $1 \mathrm{~min}$ of extension at $72^{\circ} \mathrm{C}$; and ending with a single extension of $15 \mathrm{~min}$ at $72^{\circ} \mathrm{C}$. The procedure generated a fragment of $278 \mathrm{bp}$, which was then digested with $\mathrm{HpyCH} 4 \mathrm{IV}$ at $37^{\circ} \mathrm{C}$ for $16 \mathrm{~h}$. A single 278-bp band corresponds to the wild-type $T$ homozygote; bands of 278, 179 and 99 bp stand for the TC heterozygotes; and 179 and $99 \mathrm{bp}$ for the $C$ homozygote. The DNA fragments were separated by electrophoresis on a $2.2 \%$ agarose gel and visualized by staining with ethidium bromide. Digested products $(20 \mu \mathrm{l})$ were mixed with $4 \mu \mathrm{l}$ STR $6 \mathrm{X}$ loading solution (Promega) and then run for $32 \mathrm{~min}$ at $100 \mathrm{~V}$.

Statistical analysis. Frequencies of the three genotypes among PCOS patients and controls were obtained by direct enumeration based on the electrophoresis results. The results of serum hormone levels, age, AAM and BMI are reported as the means \pm SD. Between PCOS patients and controls, age and BMI were compared using one way analysis of variance (ANOVA) and serum hormones were assessed using analysis of covariance to correct for age and BMI. Genotypic distributions between patients and controls were compared by the $\chi^{2}$ test of the $2 \times 3$ tables. Differences in serum hormone levels among individuals with different genotypes were assessed by using ANOVA. The Tukey test was used for further analysis of the differences among the three genotypes. Hardy-Weinberg distribution of genotypes in the PCOS and control groups was assessed using the $\chi^{2}$ test. $\mathrm{p}<0.05$ was considered statistically significant. Analyses were performed using SPSS version 17.0.

\section{Results}

Marked differences were revealed between PCOS patients and controls, some of which were consistent with the PCOS diagnostic criteria (Table I). The overall CYP19 rs2470152 genotypic distributions in all of the subjects were 0.212 for $T T, 0.514$ for $T C$ and 0.274 for $C C$. The genotypic distributions $(T T, T C$ and $C C)$ in women with PCOS $(0.228,0.484$ and 0.288 , respectively) did not differ from that of the controls $(0.192,0.552$ and 0.256 , respectively) $(\mathrm{p}=0.208)$ (Table II). The rs2470152 $T$ allele frequency in PCOS patients $(0.470)$ was similar to that in controls $(0.468)(p=0.949)$, as was the allele $C$. The genotypic distributions were in agreement with Hardy-Weinberg equilibrium.

Data analysis. The E2/T ratios were significantly different between the three genotypes in PCOS patients and controls ( $p=0.01036$ and $p=0.0000$, respectively) (Table III). Further analysis (Turkey-test) revealed statistical differences $(\mathrm{p}<0.05)$ between the two groups among the various genotypes (Fig. 1A). A significant difference was also found in the testosterone level between the $C C$ and $T C$ genotypes in PCOS patients (Fig. 1B). There were no statistical differences in BMI, AAM or levels of other serum hormones, such as LH, FSH and E2 (Table III), among the three genotypes of CYP19 rs2470152 in PCOS patients and controls. 
Table I. Clinical and endocrine characteristics of PCOS patients and controls.

\begin{tabular}{lccccccccc}
\hline & No. & $\begin{array}{c}\text { Age } \\
\text { (years })\end{array}$ & $\begin{array}{c}\mathrm{BMI}^{\mathrm{a}} \\
\left(\mathrm{kg} / \mathrm{m}^{2}\right)\end{array}$ & $\begin{array}{c}\text { FSH } \\
(\mathrm{IU} / \mathrm{l})\end{array}$ & $\begin{array}{c}\mathrm{LH}^{\mathrm{a}} \\
(\mathrm{IU} / \mathrm{l})\end{array}$ & $\begin{array}{c}\mathrm{LH} / \mathrm{FSH}^{\mathrm{a}} \\
(\mathrm{pg} / \mathrm{ml})\end{array}$ & $\begin{array}{c}\mathrm{E}^{\mathrm{a}} \\
(\mathrm{nmol} / \mathrm{l})\end{array}$ & $\begin{array}{c}\mathrm{T}^{\mathrm{a}} \\
(\mathrm{years})\end{array}$ \\
\hline Control & 297 & $31.77 \pm 4.70$ & $21.44 \pm 3.68$ & $9.76 \pm 8.70$ & $5.80 \pm 6.86$ & $0.78 \pm 1.05$ & $43.43 \pm 23.88$ & $1.48 \pm 1.56$ & $14.71 \pm 1.36$ \\
PCOS & 364 & $25.93 \pm 4.78$ & $22.57 \pm 3.81$ & $10.20 \pm 8.66$ & $17.06 \pm 7.02$ & $2.50 \pm 1.07$ & $51.19 \pm 24.06$ & $4.14 \pm 4.38$ & $14.27 \pm 1.90$ \\
\hline
\end{tabular}

BMI, body mass index; FSH, follicle-stimulating hormone; LH, luteinizing hormone; E2, estradiol; T, testosterone; AAM, age at menarche. ${ }^{\mathrm{a}} \mathrm{p}<0.05$ between PCOS group and control group.

Table II. Frequency distribution of CYP19 rs2470152 in women with PCOS and controls.

\begin{tabular}{|c|c|c|c|c|c|c|c|}
\hline \multirow[t]{2}{*}{ rs2470152 } & \multicolumn{3}{|c|}{ Genotypes, n (\%) } & \multirow[t]{2}{*}{$\mathrm{p}$-value ${ }^{\mathrm{a}}$} & \multicolumn{2}{|c|}{ Alleles, n (\%) } & \multirow[t]{2}{*}{$\mathrm{p}$-value } \\
\hline & $C C$ & $T C$ & $T T$ & & $C$ & $T$ & \\
\hline Control & $76(0.256)$ & $164(0.552)$ & $57(0.192)$ & 0.208 & $316(0.532)$ & $278(0.468)$ & 0.949 \\
\hline PCOS & $105(0.288)$ & $176(0.484)$ & $83(0.228)$ & & $386(0.530)$ & $342(0.470)$ & \\
\hline
\end{tabular}

${ }^{a}$ Based on the genotype frequencies vs. control. ${ }^{b}$ Based on the allele frequencies vs. control.

Table III. Anthropometric characteristics and serum hormone concentrations in different genotypic women with PCOS and controls.

\begin{tabular}{|c|c|c|c|c|c|c|c|c|}
\hline \multirow[b]{2}{*}{ Genotypes } & \multicolumn{3}{|c|}{ Control } & \multirow[t]{2}{*}{ p-value } & \multicolumn{3}{|c|}{ PCOS } & \multirow[t]{2}{*}{ p-value } \\
\hline & $C C$ & $T C$ & $T T$ & & $C C$ & $T C$ & $T T$ & \\
\hline Age (years) & $32.35 \pm 5.12$ & $31.61 \pm 4.61$ & $31.48 \pm 4.40$ & 0.50710 & $23.37 \pm 5.75$ & $25.80 \pm 4.22$ & $26.88 \pm 4.54$ & 0.16050 \\
\hline AAM (years) & $14.92 \pm 1.85$ & $14.54 \pm 1.00$ & $15.25 \pm 1.90$ & 0.50413 & $14.31 \pm 2.83$ & $14.37 \pm 1.84$ & $14.41 \pm 1.51$ & 0.96918 \\
\hline BMI $\left(\mathrm{kg} / \mathrm{m}^{2}\right)$ & $21.35 \pm 3.30$ & $21.54 \pm 4.16$ & $21.29 \pm 2.58$ & 0.89340 & $22.74 \pm 3.79$ & $22.57 \pm 3.73$ & $22.35 \pm 4.06$ & 0.83184 \\
\hline FSH (IU/1) & $8.50 \pm 4.66$ & $8.07 \pm 2.63$ & $8.36 \pm 3.24$ & 0.68299 & $7.32 \pm 3.37$ & $7.68 \pm 4.14$ & $8.91 \pm 4.47$ & 0.21618 \\
\hline LH (IU/l) & $4.96 \pm 2.62$ & $5.35 \pm 3.26$ & $5.93 \pm 4.13$ & 0.32229 & $17.94 \pm 21.83$ & $20.11 \pm 19.46$ & $22.22 \pm 10.22$ & 0.59369 \\
\hline LH/FSH & $0.64 \pm 0.35$ & $0.70 \pm 0.45$ & $0.75 \pm 0.49$ & 0.46051 & $2.40 \pm 1.32$ & $2.61 \pm 1.54$ & $2.87 \pm 1.54$ & 0.35264 \\
\hline $\mathrm{T}(\mathrm{nmol} / \mathrm{l})$ & $1.30 \pm 1.04$ & $1.22 \pm 0.69$ & $1.30 \pm 0.65$ & 0.94452 & $3.10 \pm 2.20$ & $4.60 \pm 4.24$ & $3.40 \pm 2.20$ & 0.00625 \\
\hline $\mathrm{E} 2(\mathrm{pg} / \mathrm{ml})$ & $40.87 \pm 18.69$ & $45.40 \pm 22.66$ & $50.19 \pm 23.02$ & 0.12189 & $60.53 \pm 33.55$ & $58.14 \pm 28.11$ & $67.51 \pm 28.81$ & 0.17173 \\
\hline $\mathrm{E} 2 / \mathrm{T}(\ln )$ & $4.77 \pm 0.79$ & $3.05 \pm 0.79$ & $4.63 \pm 1.31$ & $4.69 \mathrm{E}-7$ & $4.42 \pm 0.74$ & $3.97 \pm 0.85$ & $4.30 \pm 0.89$ & 0.01036 \\
\hline $\operatorname{PRL}(\mu \mathrm{g} / \mathrm{l})$ & $59.10 \pm 110.6$ & $16.35 \pm 8.64$ & $15.20 \pm 10.10$ & 0.10368 & $18.01 \pm 13.79$ & $24.08 \pm 37.19$ & $16.75 \pm 16.49$ & 0.21401 \\
\hline
\end{tabular}

AAM, age at menarche; BMI, body mass index; FSH, follicle-stimulating hormone; LH, luteinizing hormone; T, testosterone; E2, estradiol; E2/T, estradiol/testosterone ratio; PRL, prolactin.

\section{Discussion}

The CYP19 localized at chromosome 15q21.1 encodes aromatase, which is a key enzyme for estrogen biosynthesis (20). CYP19 variations may affect steriodogenesis in the ovary and adrenal glands. As a result, steroid hormone levels may be impacted, which in turn may affect steroid-related phenotypes. Several SNPs in CYP19 have been reported to be associated with aromatase activity. In a 684-sample study Jin et al reported that rs2414096 in CYP19 was associated with PCOS and that the $A$ allele was positively associated with aromatase activity $(\mathrm{p}=0.04)(8)$; Wang et al conducted a large case-control study involving 1,078 samples and found that the $\mathrm{Arg}^{214}$ Cys variant of CYP19 (rs700519) was associated with PCOS ( $\mathrm{p}=0.004)$. In an additional functional study, they found that 293 embryonic kidney cells transfected with the $\mathrm{Arg}^{264} \mathrm{Cys}$ variant (minor $T$ allele) resulted in increased conversion of androstenedione to estrogen when compared to WT (major $C$ allele) construct $(\mathrm{p}<0.001)(21)$. Meanwhile, Jiang et al carried out a 1,402-sample study and found that rs2470152 and rs2899470 were associated with aromatase activity $(\mathrm{p}=0.023$ and $\mathrm{p}<0.001$, respectively) (17). These studies indicate that SNPs in CYP19 may change the activity of aromatase, and thus may lead to PCOS. 
A

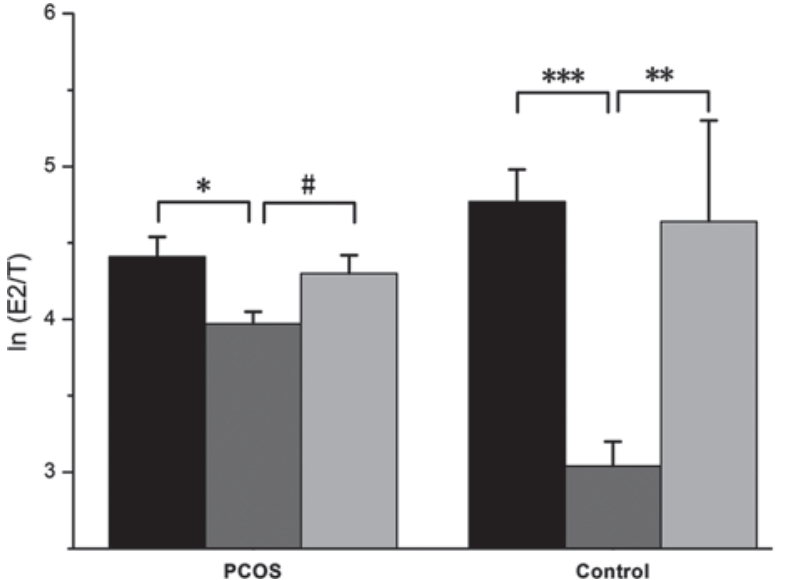

B

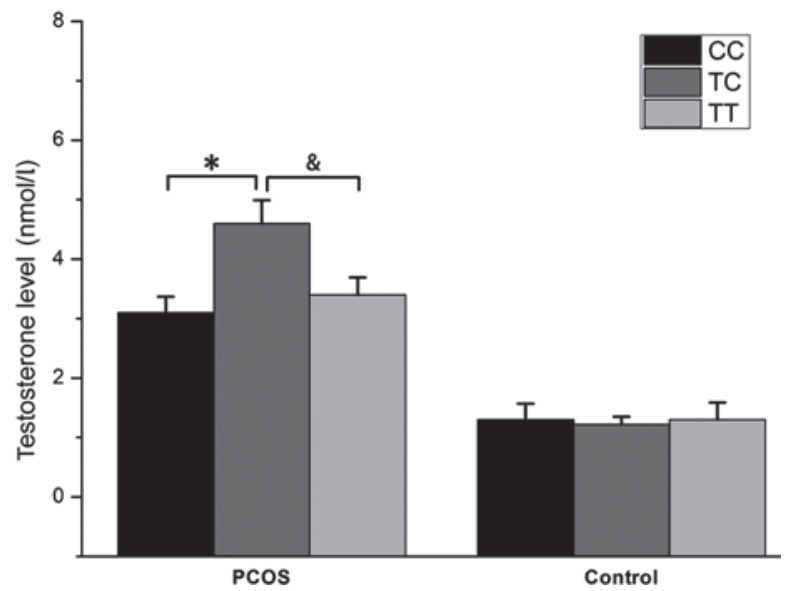

Figure 1. (A) Serum E2/T ratio and (B) testosterone level of PCOS patients and controls according to their rs 2470152 polymorphism genotypes. For the PCOS group, a total of 275 patient steroid levels were available $(82,131$ and 62 subjects with the $C C, T C$ and $T T$ genotypes, respectively). In the healthy controls, 235 were available $\left(60,127\right.$ and 48 subjects with the $C C, T C$ and $T T$ genotypes, respectively). Each bar was reported as the means \pm SEM. ${ }^{* * *}$ p $<0.001,{ }^{* *}$ p $<0.005$ and ${ }^{*} \mathrm{p}<0.05 .{ }^{\#} \mathrm{p}=0.055,{ }^{\&} \mathrm{p}=0.063$ vs. subjects with the $T C$ genotype. All p-values are compared vs. the subjects with the $T C$ genotype.

CYP19 rs2470152 is located in intron 2 and thus does not change the amino acid sequences of aromatase. However, variations in the introns may affect regulatory sequences in close proximity. CYP19 is composed of a $30-\mathrm{kb}$ coding region and a $93-\mathrm{kb}$ regulatory region, the latter containing at least 10 distinct tissue-specific promoters. The rs 2470152 is located in the region of the I.4 promoter (22). Since the conversion of androgens (T) to estrogens (E2) is catalyzed by aromatase, the E2/T ratio may be a marker of aromatase activity with a positive correlation. In our observation, E2/T was significantly different among the three genotypes (Fig. 1A). Notably, in PCOS patients and controls, E2/T was lower in the heterozygous $T C$ genotype than the other two homozygous genotypes, although it was not significantly different between the $T T$ and $T C$ genotypes in PCOS patients $(\mathrm{p}=0.05477)$. This may be due to a strong linkage disequilibrium with other functional variants in exons, resulting in the change of protein sequence of aromatase, which may cause a dominant-negative effect of aromatase activity in the heterozygous genotype. Aromatase activity was lower in the heterozygous $T C$ genotype of rs2470152, leading to hyperandrogenism in this genotype (Table III). In our findings, the SNP rs2470152 C/T polymorphisms were correlated to aromatase activity. This may be explained by the hypothesis of Sofe et al that the rs 2470152 variation alters a potential binding site for the transcription factor cAMP response element binding protein, which is crucial to the aromatase expression regulation (23).

The estradiol level was not different between the three genotypes in PCOS patients and controls. These may be due to the negative feedback regulation of the hypothalamic-pituitaryovarian axis. Testosterone levels were higher in the TC genotype than the other two genotypes in PCOS patients, which may be the result of the lower activity of aromatase in the heterozygous $T C$ genotype mentioned above, given that the testosterone level was high enough in the PCOS patients. In the controls, testosterone levels were similar among the three genotypes. The mechanism may be accounted for by the fact that the aromatase activity was high in the $C C$ and $T C$ genotypes, whereas the zymolyte (testosterone) level was not high enough to sustain the conversion rate in controls, resulting in similar testosterone levels in the three genotypes. Therefore, PCOS patients with hyperandrogenism may be a model with which to study the aromatase activity among different genotypes.

In the Gothenburg Osteoporosis and Obesity Determinants (GOOD) project studying young adult men, Eriksson et al demonstrated that subjects with the $C C$ genotype of rs 2470152 in CYP19 had 13\% higher E2 levels than those with the TT genotype (16). Jiang et al also found that the E2/T ratio was significantly higher in the $C C$ genotype compared to the $T T$ genotype of rs2470152 in Osteoporotic Fractures in Men (MrOS) patients (17). In our study, we failed to demonstrate differences in the E2 levels between the three genotypes of rs2470152, nor did we find any difference in the E2/T ratio between the $C C$ and $T T$ genotypes in PCOS patients and controls. Several reasons may account for the discrepancies. Firstly, the selection criteria for the subjects were different. i) Subjects in the study of Eriksson et al were of Caucasian origin, while in our study they were all Han Chinese; in addition, the allele frequencies of SNP rs2470152 were not the same between the two ethnic groups. ii) Eriksson et al chose adult young men, Jiang et al selected elderly Chinese men with MrOS and we examined young Chinese women with PCOS. The genetic and metabolic factors are different between men and women to a certain extent, as well as among people with different diseases. Secondly, other unknown SNPs in CYP19 or other related genes may have a potential effect on steriodogenesis and they could affect both testosterone and estrogen levels; the allelic frequencies of these SNPs also vary within the two ethnic groups (17).

In conclusion, to our knowledge, SNP rs2470152 in CYP19 was not a major etiological factor for PCOS, but the heterozygous $T C$ genotype may inhibit aromatase activity and thus result in hyperandrogenism, particularly in PCOS patients.

\section{Acknowledgements}

The authors are extremely grateful to all the women who participated in the study. This study was supported by the National Natural Science Foundation of China (30672228) and the Natural Basic Research Program of China (973 program 2010CB945103). 


\section{References}

1. Wood JR, Nelson VL, Ho C, et al: The molecular phenotype of polycystic ovary syndrome (PCOS) theca cells and new candidate PCOS genes defined by microarray analysis. J Biol Chem 278: 26380-26390, 2003

2. Azziz R, Carmina E, Dewailly D, et al: Positions statement: criteria for defining polycystic ovary syndrome as a predominantly hyperandrogenic syndrome: an Androgen Excess Society guideline. J Clin Endocrinol Metab 91: 4237-4245, 2006.

3. Diamanti-Kandarakis E: Polycystic ovarian syndrome: pathophysiology, molecular aspects and clinical implications. Exp Rev Mol Med 10: e3, 2008.

4. Dunaif A: Insulin resistance and the polycystic ovary syndrome: mechanism and implications for pathogenesis. Endocr Rev 18 774-800, 1997.

5. Yildiz BO, Yarali H, Oguz H and Bayraktar M: Glucose intolerance, insulin resistance, and hyperandrogenemia in first degree relatives of women with polycystic ovary syndrome. J Clin Endocrinol Metab 88: 2031-2036, 2003.

6. Williamson K, Gunn AJ, Johnson N and Milsom SR: The impact of ethnicity on the presentation of polycystic ovarian syndrome. Aust N Z J Obstet Gynaecol 41: 202-206, 2001.

7. Jakimiuk AJ, Weitsman SR, Brzechffa PR and Magoffin DA Aromatase mRNA expression in individual follicles from polycystic ovaries. Mol Hum Reprod 4: 1-8, 1998.

8. Jin JL, Sun J, Ge HJ, et al: Association between CYP19 gene SNP rs2414096 polymorphism and polycystic ovary syndrome in Chinese women. BMC Med Genet 10: 139, 2009.

9. Gharani N, Waterworth DM, Batty S, et al: Association of the steroid synthesis gene CYP1la with polycystic ovary syndrome and hyperandrogenism. Hum Mol Genet 6: 397-402, 1997.

10. Carey AH, Waterworth D, Patel K, et al: Polycystic ovaries and premature male pattern baldness are associated with one allele of the steroid metabolism gene CYP17. Hum Mol Genet 3: 1873-1876, 1994.

11. Jones MR, Italiano L, Wilson SG, et al: Polymorphism in HSD17B6 is associated with key features of polycystic ovary syndrome. Fertil Steril 86: 1438-1446, 2006.

12. Ferk P, Perme MP and Gersak K: Insulin gene polymorphism in women with polycystic ovary syndrome. J Int Med Res 36 $1180-1187,2008$
13. Xu P, Che Y, Cao Y, et al: Polymorphisms of TCF7L2 and HHEX genes in Chinese women with polycystic ovary syndrome. J Assist Reprod Genet 27: 23-28, 2010.

14. Chen SA, Besman MJ, Sparkes RS, et al: Human aromatase: cDNA cloning, Southern blot analysis, and assignment of the gene to chromosome 15. DNA 7: 27-38, 1988.

15. Harada N, Ogawa H, Shozu M and Yamada K: Genetic studies to characterize the origin of the mutation in placental aromatase deficiency. Am J Hum Genet 51: 666-672, 1992.

16. Eriksson AL, Lorentzon M, Vandenput L, et al: Genetic variations in sex steroid-related genes as predictors of serum estrogen levels in men. J Clin Endocrinol Metab 94: 1033-1041, 2009.

17. Jiang J, Tang NL, Ohlsson C, et al: Association of genetic variations in aromatase gene with serum estrogen and estrogen/testosterone ratio in Chinese elderly men. Clin Chim Acta 411: 53-58, 2010

18. Rotterdam ESHRE/ASRM-Sponsored PCOS Consensus Workshop Group: Revised 2003 consensus on diagnostic criteria and long-term health risks related to polycystic ovary syndrome. Fertil Steril 81: 19-25, 2004.

19. Sun J, Fan H, Che Y, et al: Association between ACE gene I/D polymorphisms and hyperandrogenism in women with polycystic ovary syndrome (PCOS) and controls. BMC Med Genet 10: 64 2009.

20. Bulun SE, Takayama K, Suzuki T, Sasano H, Yilmaz B and Sebastian S: Organization of the human aromatase p450 (CYP19) gene. Sem Reprod Med 22: 5-9, 2004.

21. Wang $\mathrm{H}, \mathrm{Li} \mathrm{Q}$, Wang $\mathrm{T}$, et al: A common polymorphism in the human aromatase gene alters the risk for polycystic ovary syndrome and modifies aromatase activity in vitro. Mol Hum Reprod 17: 386-391, 2011.

22. Bulun SE, Sebastian S, Takayama K, Suzuki T, Sasano H and Shozu M: The human CYP19 (aromatase P450) gene: update on physiologic roles and genomic organization of promoters. J Ster Biochem Mol Biol 86: 219-224, 2003.

23. Sofi M, Young MJ, Papamakarios T, Simpson ER and Clyne CD: Role of CRE-binding protein (CREB) in aromatase expression in breast adipose. Breast Cancer Res Treat 79: 399-407, 2003. 